\title{
In vivo color flow mapping using synthetic aperture dual stage beamforming
}

\author{
Li, Ye; Hemmsen, Martin Christian; Nielsen, Michael Bachmann; Jensen, Jørgen Arendt
}

Published in:

Proceedings of IEEE International Ultrasonics Symposium

Link to article, DOI:

10.1109/ULTSYM.2012.0398

Publication date:

2012

Document Version

Early version, also known as pre-print

Link back to DTU Orbit

Citation (APA):

Li, Y., Hemmsen, M. C., Nielsen, M. B., \& Jensen, J. A. (2012). In vivo color flow mapping using synthetic aperture dual stage beamforming. In Proceedings of IEEE International Ultrasonics Symposium (pp. 1592-1595). IEEE. https://doi.org/10.1109/ULTSYM.2012.0398

\section{General rights}

Copyright and moral rights for the publications made accessible in the public portal are retained by the authors and/or other copyright owners and it is a condition of accessing publications that users recognise and abide by the legal requirements associated with these rights.

- Users may download and print one copy of any publication from the public portal for the purpose of private study or research.

- You may not further distribute the material or use it for any profit-making activity or commercial gain

- You may freely distribute the URL identifying the publication in the public portal 
Paper presented at the IEEE International Ultrasonics Symposium, Dresden, Germany, 2012:

\section{In vivo color flow mapping using synthetic aperture dual stage beamforming}

Ye Li ${ }^{1}$, Peter Moller Hansen ${ }^{1,2}$, Martin Christian Hemmsen ${ }^{1}$, Michael Bachmann Nielsen ${ }^{2}$, Jorgen Arendt Jensen ${ }^{1}$

${ }^{1}$ Center for Fast Ultrasound Imaging,

Biomedical Engineering group, Department of Electrical Engineering, Bldg. 349,

Technical University of Denmark, DK-2800 Kgs. Lyngby, Denmark

${ }^{2}$ Department of Radiology, Rigshospitalet, 2100 Copenhagen, Denmark, 


\title{
In vivo color flow mapping using synthetic aperture dual stage beamforming
}

\author{
Ye Li ${ }^{1}$, Peter Møller Hansen ${ }^{1,2}$, Martin Christian Hemmsen ${ }^{1}$, Michael Bachmann Nielsen ${ }^{2}$, Jørgen Arendt Jensen ${ }^{1}$ \\ ${ }^{1}$ Center for Fast Ultrasound Imaging, Department of Electrical Engineering, Build. 349 \\ Technical University of Denmark, 2800, Kgs. Lyngby, Denmark \\ ${ }^{2}$ Department of Radiology, Rigshospitalet, 2100 Copenhagen, Denmark
}

\begin{abstract}
An in vivo investigation of synthetic aperture flow imaging using a dual stage beamformer is presented in this paper. In the previous work, simulations and Doppler flow phantom experiments showed promising results, which indicated the methods capability of producing fast color flow mapping with a good quality. Due to the continuous data, both high velocity and low velocity can be estimated. Moreover, synthetic aperture flow imaging can be implemented on a commercial platform, because the number of calculations have been reduced. In this work, A commercial ultrasound scanner (Pro Focus equipped with a UA 2227 Research Interface, BK Medical, Herlev, Denmark) was used to transmit signals and record echoes. The data are processed off-line. The method is validated using a pulsatile flow phantom. Volume flow is calculated, and is compared with the volume flow set for the pump. The relative standard deviation is $14.3 \%$ and relative bias is $6.4 \%$ for the phantom measurements. The blood flow in a common carotid artery of a 35-year-old healthy male is scanned by a medical doctor (PMH). The in vivo data is processed off-line. Fast synthetic aperture color flow mapping with frame rate of $85 \mathrm{~Hz}$ is produced and the volume flow is calculated.
\end{abstract}

\section{INTRODUCTION}

Synthetic aperture sequential beamforming (SASB) has been investigated by Kortbek and Jensen [1] through simulations and phantom measurements. Hemmsen et al [2] extended SASB to convex array transducer and studied its performance on in vivo data. These demonstrates that SASB can produce ultrasound images with good penetration, lateral resolution independency, and less calculations are required compared to full synthetic aperture imaging. In our previous work [3], [4], synthetic aperture using a dual stage beamformer approach is used for flow imaging. Simulations and flow phantom experiments showed that the new method can produce fast color flow images for constant flow. The standard deviations and the bias are less than $10 \%$, if parameters are optimized.

The purpose of the research presented in this paper is to validate the dual beamformer approach approach on in vivo data. A commercial ultrasound scanner was used to produce data, which are focused using a fixed delay profile along the depth. Before the in vivo measurements, the method is validated using a pulsatile flow phantom mimicking blood flow over the cardiac cycle. Several important parameters are explained and discussed. The volume flow is calculated and compared with the waveform generated by the flow pump. The optimized setup is used for in vivo data processing. The blood flow in a common carotid artery is scanned, and the recorded signals are processed off-line.

\section{METHOD AND DATA ACQUISITION SYSTEM}

A focused ultrasound beam is transmitted by a sub-aperture, and echoes are received by the same aperture. Focusing is split into two steps. Data are only focused at virtual source in the first stage, and are focused dynamically in the second stage. The high resolution image is created by adding a number of newest low resolution images. The complete experimental setup consists of two units: the ultrasound research system and the flow circulation system. The flow circulation system consists of a blood-mimicking fluid and a PC-controlled pump (CompuFlow 1000, Shelley Medical Imaging Technologies, London, Ontario, Canada) [5]. The ultrasound research system [6] consists of a commercial ultrasound scanner (Pro Focus equipped with a UA 2227 Research Interface, BK Medical, Herlev, Denmark) and a standard PC. The beamformed signals with a fixed virtual source is recorded, and are processed offline to make a color flow image.

The basic parameters used in the experiment are shown in Table I

TABLE I

PARAMETERS FOR THE MEASUREMENT.

\begin{tabular}{lrc} 
Parameter & Value & Unit \\
\hline Central frequency & 7 & $\mathrm{MHz}$ \\
Sampling frequency & 34.28576 & $\mathrm{MHz}$ \\
Total elements & 192 & \\
Transducer pitch & 0.208 & $\mathrm{~mm}$ \\
Transducer height & 4.5 & $\mathrm{~mm}$ \\
Transmit and receive focal depth (virtual source) & 5 & $\mathrm{~mm}$ \\
F number in transmit & 0.5 & \\
F number in receive & 2 & \\
Radius of vessel & 6.4 & $\mathrm{~mm}$ \\
\hline
\end{tabular}

\section{OPTIMIZATION OF PARAMETERS}

To make a proper in vivo scanning, the estimation scheme should be reexamined and optimized. In this section, several important parameters are discussed. The optimization is made based on pulsatile flow phantom measurement, and the results are discussed as well.

1) Number of transmissions

The velocities are found by cross-correlating two images from 
the same transmission sequence. The true pulse repetition time used in the velocity estimator is dependent on the time period between the two emissions

$$
T_{p r f}^{\prime}=N \cdot T_{p r f},
$$

where $N$ is the number of emissions in one sequence and $T_{p r f}$ is the time period between two consecutive emissions.

On the other hand, the number of transmissions determine the image area. More emissions with large sparseness can generate larger images, because the apodization function applied on the LRIs, the image area at a given depth is determined by the number of emissions used for constructing a HRI. Therefore, the number of transmissions is limited to $N=4$, which balances the motion effects against the width of the images.

\section{2) The position of virtual sources}

Two points have to be considered when deciding the position of virtual sources: $a$ ) Opening angle: The relation between the opening angle $\alpha$ and $F^{\#}$ is $\alpha=2 \arctan \frac{1}{2 F^{\#} \text {. The virtual }}$ source at small depths yields a large opening angle, i.e. a large spatial span. Such setup can expand the imaging area. b) Position: Because the transmit beam is only focused at the virtual source and sparse transmission sequence is employed, the signal-to-noise ratio in the area between two consecutive virtual sources is very low. Thus, choosing its position as the same as the target should be avoided.

To make in vivo measurements possible, a 'blind area' between virtual sources should be carefully considered. For example, the carotid artery is the scanning target for the in vivo measurement. Normally, in the upper part of its course it is more superficial, being covered merely by the integument, the superficial fascia. The distance between the common carotid artery and superficial integument is very short (only $18-20 \mathrm{~mm}$ in the volunteer's neck). Therefore, it is not suitable to set the virtual source at that depth. To prevent placing the common carotid artery in the 'blind area', the virtual sources are set at $5 \mathrm{~mm}$ from the transducer.

3) Number of averaged cross-correlation functions

A number of cross-correlation functions are averaged to improve the performance of cross-correlation function. The procedure is predicted on the assumption that the cross-correlation functions have the same position of the maximum peak, i.e. the time shift remains the same over the averaging time. For a constant flow, any number of estimates can be averaged, since the velocities does not change over time. However, this will not be the case for pulsatile flow, because the velocity is a function of both time and position. There are accelerations and decelerations of blood flow in the human vessel, thus a gradual shift in the position of the peak will occur due to changing velocity. The gradual shift should be limited to no more than half a sample over the entire time duration of the averaging. A velocity change corresponding to half a sample $\operatorname{shift}(\Delta z)$ is

$$
\Delta \nu_{\frac{1}{2}}=\frac{\frac{1}{2} \Delta z}{N T_{p r f}} .
$$

It should be less than the velocity change within the averaging period. The velocity change in the blood vessel is defined as $a \cdot T_{\text {avg }}=a \cdot N_{c} \cdot T_{p r f}$, where $a$ is the acceleration of the blood flow

$$
a=\frac{\text { peak velocity }- \text { low velocity }}{\text { pulse rise time }} .
$$

Fig. 1 illustrates how the values are measured from a velocity waveform. After reading values from the figure, the acceleration of the flow is approximately $2.4 \mathrm{~m} / \mathrm{s}^{2}$ in the carotid artery. The relation requires that

$$
a \cdot N_{c} \cdot T_{p r f}<\frac{\frac{1}{2} \Delta z}{N T_{p r f}} .
$$

For the current imaging situation, the parameters are $N=4$, $f_{s}=34.29 \mathrm{MHz}, c=1540 \mathrm{~m} / \mathrm{s}, f_{p r f}=11 \mathrm{kHz}$. It yields a maximum averaging time of $T_{\text {avg }} \approx 13 \mathrm{~ms}$, which corresponds to $N_{c}=142$.

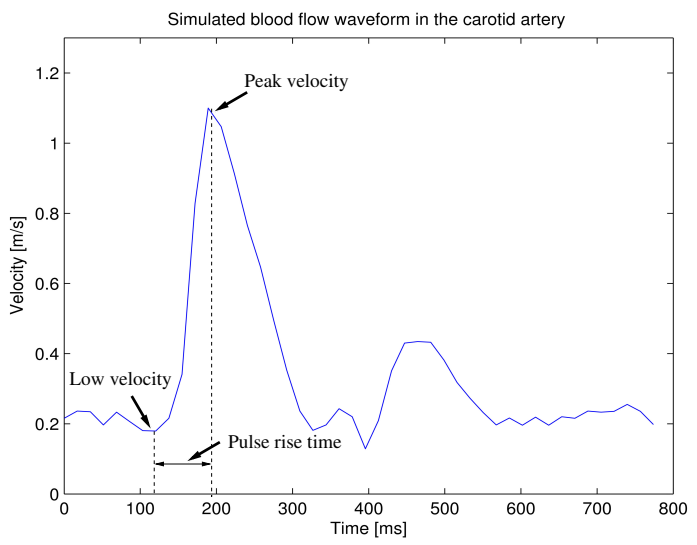

Fig. 1. Illustration of a flow waveform in a common carotid artery. The pulse rise time is defined as the period from the onset to the peak velocity. In this time period, the rate of velocity change is largest. (To average crosscorrelation functions that have the same maximum peak, the velocity change over the averaging time should be less than that of half a sample shift.)

\section{4) Echo canceling}

In ultrasound flow measurements, the blood signals are corrupted with clutter signals from muscular tissue, vessel walls etc, which are much stronger than the blood signals [8]. The purpose of echo canceling is to remove the strong echo signals from the stationary or slowly moving tissues. If the stationary signals are not suppressed, they are strong enough to mask the weak echoes from blood, making it impossible to estimate velocities from them.

In this work, a simple mean subtraction algorithm is used to remove the stationary signals. The mean value of the slow-time signal is subtracted from each sample in the signal. The images input to the echo canceler are from the 
same emission sequence, i.e. from $n+k \cdot N$, where $k$ is an integer. Images from different sequence can give rise to more decorrelations and the operations on them can introduce errors. In the in vivo measurements, the echo canceling is applied after the beamformation and 16 32 HRIs are chosen to calculate the mean value. Willemetz et al [9] reported that delay-line cancellers lead to biased estimates of frequency at low signal-to-noise ratios, however other more advanced approaches have been investigated [10], [11]. Since the HRIs are continuously available, no initialization of the filter is necessary, which can make echo canceling very efficient.

5) Intensity measurements

To make in vivo measurements, the derated global maximum acoustic output has to be measured, and should not exceed FDA acoustic output exposure levels (see Table II) [12]. Spatial-peak temporal-average intensity $I_{\text {spta }}$, spatial-peak pulse-average intensity $I_{\text {sppa }}$, and mechanical index have all been measured. All measurements are derated by an attenuation of $0.3 \mathrm{~dB} / \mathrm{cm}-\mathrm{MHz}$.

The acoustic outputs were measured in a water tank using a high precision acoustic intensity measurement system (AIMS III with Soniq 5.0 software) and a HGL0400 hydrophone (Onda, Sunnyvale, CA, USA). Table II shows that the transmission used invivo are within the FDA limits.

TABLE II

PREAMENDMENTS ACOUSTIC OUTPUT EXPOSURE

\begin{tabular}{c|c|c|c} 
Use & $I_{S P T A .3}\left(\mathrm{~mW} / \mathrm{cm}^{2}\right)$ & $I_{S P P A .3}\left(\mathrm{~W} / \mathrm{cm}^{2}\right)$ & $M I$ \\
\hline Peripheral vessel & 720 & 190 & 1.9 \\
\hline Actual value & 609 & 145 & 0.94 \\
\hline
\end{tabular}

\section{PULSATILE PHANTOM MEASUREMENTS}

The flow phantom generates a pulsatile waveform mimicking the blood flow in a common carotid artery. A commercial scanner is used for transmit and receive ultrasound signals. The recorded echoes are processed off-line. The estimated volume flow is compared to the flow produced by the flow pump. The instantaneous flow is defined as

$$
Q=\pi \sum_{n=1}^{K} r_{n} \Delta r \nu_{n}
$$

where $r_{n}$ is the distance of the semi-annulus from the centroid, $\Delta r$ is the size of the uniformly spaced sample volumes, and $\nu_{n}$ is the angle corrected velocity in a segment. The result is shown in Fig. 2, where the estimated volume flow is compared with the actual volume flow produced by the flow pump. The standard deviation is $1.43 \mathrm{ml} / \mathrm{s}$, and the mean bias is $0.64 \mathrm{ml} / \mathrm{s}$ corresponding to a relative standard deviation of $14.3 \%$ and a mean relative bias of $6.4 \%$ comparing with the peak volume flow of $10 \mathrm{ml} / \mathrm{s}$ in the pulsatile period.

The main difference is the shift of the curve in the systolic period. Since the reference waveform is the ideal output of the flow pump, the actual measurement is also dependent on the geometry of the vessel. The flow phantom used in the

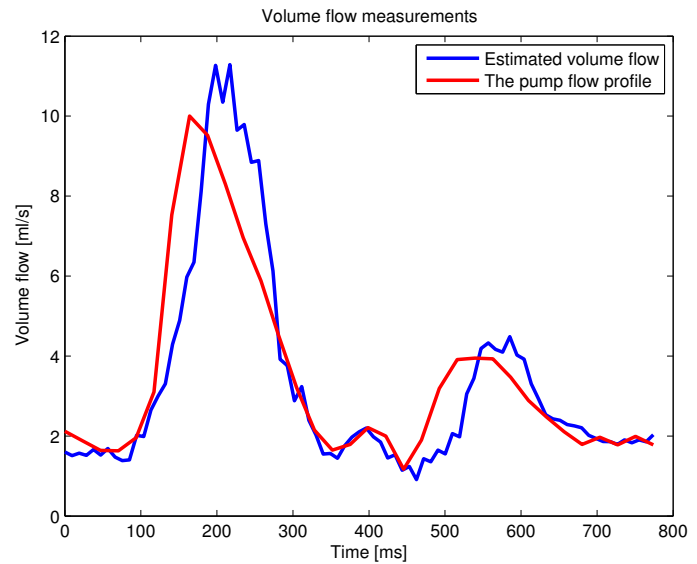

Fig. 2. The estimated volume flow for the flow circulation experiment compared with the expected flow profile produced by the flow pump. The complete time period of the pulsatile flow in the carotid artery is $774 \mathrm{~ms}$.

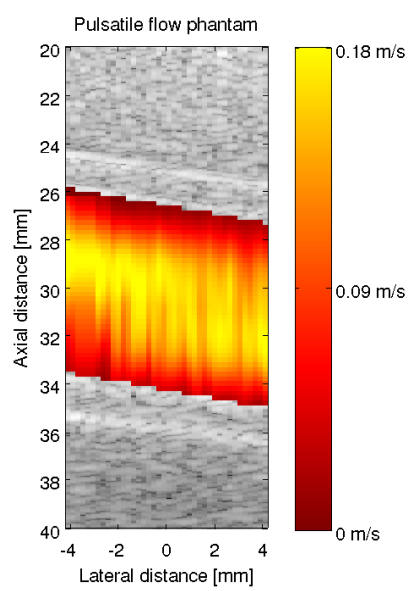

Fig. 3. Color flow mapping of the flow circulation experiment. The flowto-beam angle is 79 degrees measured from the B-mode image. The image is captured at $264 \mathrm{~ms}$.

experiment contains a straight tube connecting to a bifurcating tube, and the measurements are made at the place, which is $7.5 \mathrm{~cm}$ from the start of the bifurcation. It could be a factor influencing the final flow profile and causing the difference. Another concern for the shift is that all calculations are made under a assumption of symmetrical flow profile. Errors can occur if the flow exhibits skewing in the scan plane, i.e. when the peak velocity is not in the geometric center of the vessel.

\section{IN VIVO MEASUREMENTS}

A common carotid artery of a healthy 35 year old male was scanned by a medical doctor (PMH). The virtual source is set to $5 \mathrm{~mm}$ and 2 cycle ultrasound pulses were emitted with a center frequency of $7 \mathrm{MHz}$. The pulse repetion frequency is set to $11 \mathrm{kHz}$, and approximately 2 seconds of data are acquired corresponding to 22,000 echo signals. The received signals are convolved with a match filter, and then reconstructed in a two-stage beamformer to form HRIs, and finally the cross- 


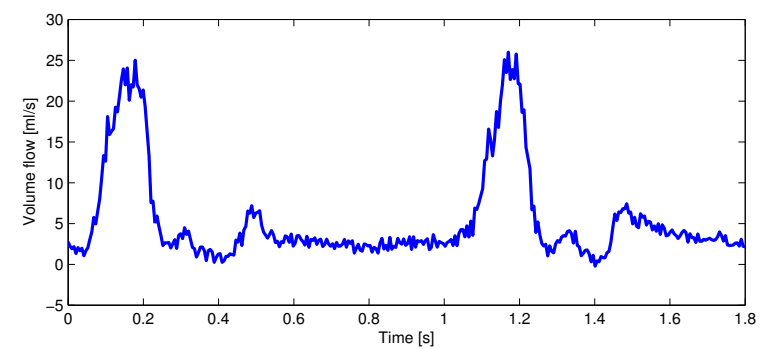

Fig. 4. The estimated volume flow for the in vivo measurement.

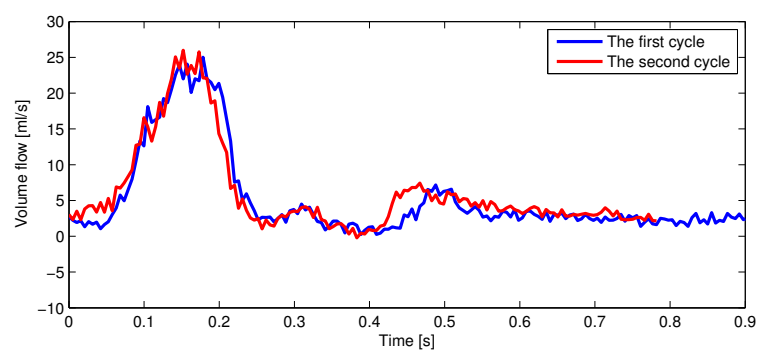

Fig. 5. Volume flow for two cardiac cycles plotted on top of each other.
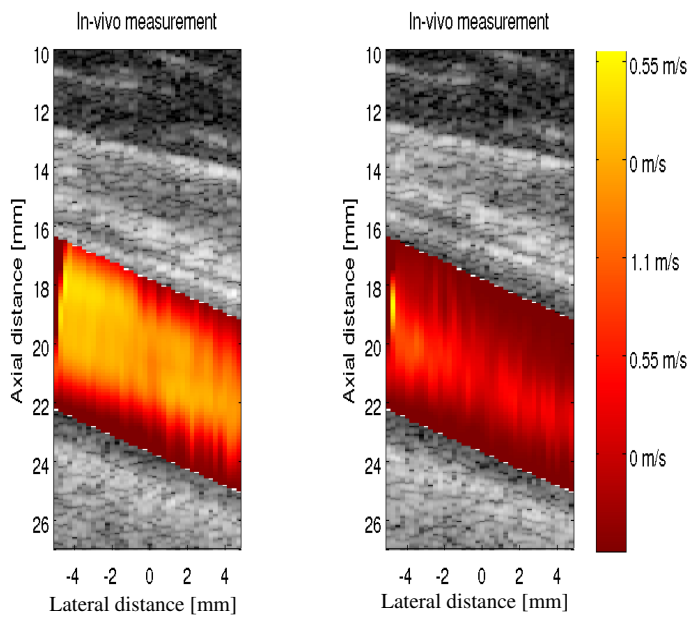

Fig. 6. Color flow mapping of the flow circulation experiment. The flowto-beam angle is 73.4 degrees measured from the B-mode image. The left image is captured at $230 \mathrm{~ms}$, and the right is captured at $650 \mathrm{~ms}$.

correlation technique is applied on these HRIs. Velocities are found using 30 sequences corresponding to 120 LRIs, and echo canceling is made by subtracting the mean value.

Fig. 4 illustrates the estimated volume flow as a function of time. Fig. 5 shows that two consecutive cardiac cycles are consistent over time. Fig. 6 show the in vivo color flow mapping. The diameter of the blood vessel is approximately 6 $\mathrm{mm}$ depending on where in the cardiac cycle it is measured. The geometry of the blood vessel is assumed as a cylinder with a symmetric cross section. The maximum value is 26.3 $\mathrm{ml} / \mathrm{s}$. The curve indicates that the volume flow is positive at all times during the scan, which is characteristic for the flow in the common carotid artery.

\section{DISCUSSION AND CONCLUSION}

The new method has been evaluated on a flow phantom, which can generate pulsatile flow waveform. The volume flow is calculated to quantify the performance. A relative standard deviation of $14.3 \%$ and bias of $6.4 \%$ are achieved using the optimized measurement setup. In the flow phantom experiment, the method is further investigated to obtain a set of reasonable measurement parameters. The new method has been tested in vivo on the blood flow of the common carotid artery of a healthy 35 years old male. Color flow mapping is calculated for visualizing the blood in the vessel and volume flow is calculated to see the cardiac cycles.

\section{ACKNOWLEDGE}

This work was supported by grant 024-2008-3 from the Danish Advanced Technology Foundation and BK Medical Aps, Denmark.

\section{REFERENCES}

[1] J. Kortbek and J. A. Jensen, "Synthetic aperture sequential beamforming," in Proc. IEEE Ultrason. Symp., pp. 966-969, 2008.

[2] M. C. Hemmsen, P. M. Hansen, T. Lange, J. M. Hansen, L. H. Kristoffer, M. B. Nielsen, and J. A. Jensen, "In vivo evaluation of synthetic aperture sequential beamforming," Ultrasound Med. Biol., vol. 38, pp. 708-716, 2012.

[3] Y. Li and J. A. Jensen, "Synthetic aperture flow imaging using a dual stage beamformer approach," in Proc. IEEE Ultrason. Symp., pp. 1924 1927, 2010.

[4] Y. Li and J. A. Jensen, "Preliminary experimental verification of synthetic aperture flow imaging using a dual stage beamformer approach," Med. Biol. Eng. Comp., vol. 15th Nordic-Baltic Conference on Biomedical Imaging, pp. 53-56, 2011.

[5] K. V. Ramnarine, D. K. Nassiri, P. R. Hoskins, and J. Lubbers, "Validation of a new blood mimicking fluid for use in Doppler flow test objects," Ultrasound Med. Biol., vol. 24, pp. 451-459, 1998.

[6] M. C. Hemmsen, S. Nikolov, M. M. Pedersen, M. J. Pihl, M. S. Enevoldsen, J. M. Hansen, and J. A. Jensen, "Implementation of a versatile research data acquisition system using a commercially available medical ultrasound scanner," IEEE Trans. Ultrason., Ferroelec., Freq. Contr., vol. 59, pp. 1487-1499, 2012.

[7] C. R. Hazard and G. R. Lockwood, "Effects of motion artifacts on a synthetic aperture beamformer for real-time 3D ultrasound," in Proc. IEEE Ultrason. Symp., pp. 1221-1224, 1999.

[8] H. Torp, "Clutter Rejection Filters in Color Flow Imaging: A Theoretical Approach," IEEE Trans. Ultrason., Ferroelec., Freq. Contr., vol. 44, no. 2, pp. 417-424, 1997.

[9] J. C. Willemetz, A. Nowicki, J. J. Meister, F. D. Palma, and G. Pante, "Bias and variance in the estimation of the Doppler frequency induced by a wall motion filter," Ultrason. Imaging, vol. 11, pp. 215-225, 1989.

[10] S. Bjaerum and H. Torp, "Statistical evaluation of clutter filters in color flow imaging," Ultrasonics, vol. 38, pp. 376-380, 2000.

[11] S. Bjærum, H. Torp, and K. Kristoffersen, "Clutter filter design for ultrasound color flow imaging," IEEE Trans. Ultrason., Ferroelec., Freq. Contr., vol. 49, pp. 204-216, 2002.

[12] Information for manufacturers seeking marketing clearance of diagnostic ultrasound systems and transducers, U.S. Department of Health and Human Services, Center for Devices and Radiological Health. 2008. 